\title{
OPERATOR MACHINES ON DIRECTED GRAPHS
}

\author{
PETR HÁJEK AND RICHARD J. SMITH
}

\begin{abstract}
We show that if an infinite-dimensional Banach space $X$ has a symmetric basis then there exists a bounded, linear operator $R: X \longrightarrow X$ such that the set

$$
A=\left\{x \in X:\left\|R^{n}(x)\right\| \rightarrow \infty\right\}
$$

is non-empty and nowhere dense in $X$. Moreover, if $x \in X \backslash A$ then some subsequence of $\left(R^{n}(x)\right)_{n=1}^{\infty}$ converges weakly to $x$. This answers in the negative a recent conjecture of Prăjitură. The result can be extended to any Banach space containing an infinite-dimensional, complemented subspace with a symmetric basis; in particular, all 'classical' Banach spaces admit such an operator.
\end{abstract}

\section{INTRODUCTION}

Given a Banach space $X$, a bounded linear operator $T$ on $X$ and $x \in X$, we say that the orbit of $x$ with respect to $T$ is the set

$$
\operatorname{orb}(x, T)=\left\{T^{n}(x): n \geq 0\right\} .
$$

It is well known (cf [8]) that if $X$ is finite-dimensional then $\operatorname{orb}(x, T)$ is 'regular', in the sense that either $\left\|T^{n}(x)\right\| \rightarrow 0,\left\|T^{n}(x)\right\| \rightarrow \infty$, or there exists $M>0$ such that $M^{-1} \leq\left\|T^{n}(x)\right\| \leq M$ for all $n$. The infinite-dimensional situation is very different. Rolewicz provided simple examples of operators on infinite-dimensional spaces which admit hypercyclic vectors, that is, vectors with norm-dense orbits.

The study of orbits is connected to the invariant subspace problem. Indeed, an operator $T$ on a Banach space $X$ has no non-trivial, closed, invariant subspaces if and only if $\operatorname{span} \operatorname{orb}(x, T)$ is norm-dense for every non-zero $x \in X$.

There is a considerable body of literature on operators which admit hypercyclic vectors. In this note, we study operators with more regular orbits, in particular, those which tend to infinity. This type of orbit has received attention from several authors. For example, in a systematic study of orbits of operators on Hilbert space, Beauzamy provides several sufficient conditions for $T$ to admit a norm-dense set of points $x$ satisfying $\left\|T^{n}(x)\right\| \rightarrow \infty$ [2, Chapter III]. Broadly speaking, these conditions are based on the growth of the sequence $\left(\left\|T^{n}\right\|\right)_{n=1}^{\infty}$. For example, if $\sum_{n=1}^{\infty}\left\|T^{n}\right\|^{-1}<\infty$ then $T$ admits such a dense set. Sharp estimates of this nature applying to general Banach spaces are given in [6]. We refer the reader to the surveys [4, 5] for additional results on this topic.

Date: November 20, 2018.

2000 Mathematics Subject Classification. $47 \mathrm{~A} 05$.

Key words and phrases. Orbits of operators.

Both authors are supported by Grant A 100190801 and Institutional Research Plan AV0Z10190503. 
A given operator can have both regular and highly irregular orbits, and the exact behaviour of $\operatorname{orb}(x, T)$, as $x$ ranges over $X$, is not so easy to determine. In [7], Prăjitură makes the following conjecture.

Conjecture 1.1 ([7, Conjecture 2.9]). Let $T$ be an operator $T$ on a Banach space and let

$$
A_{T}=\left\{x \in X:\left\|T^{n}(x)\right\| \rightarrow \infty\right\} .
$$

Then $A_{T}$ is norm-dense whenever $A_{T}$ is non-empty.

Of course, if $\left\|T^{n}(x)\right\| \rightarrow \infty$ for some $x$ then $\left(\left\|T^{n}\right\|\right)_{n=1}^{\infty}$ is unbounded, so by the uniform boundedness principle, the set of $y$ with the property that $\sup _{n}\left\|T^{n}(y)\right\|=$ $\infty$ is a norm-dense $G_{\delta}$ in $X$. However, this clearly does not say anything about whether $\left\|T^{n}(y)\right\|$ tends to infinity or not. Indeed, the weighted backwards shift operator $T$ on $\ell_{p}, 1 \leq p<\infty$, given by

$$
T\left(e_{i}\right)= \begin{cases}(i /(i-1))^{\frac{1}{p}} e_{i-1} & \text { if } i>1 \\ 0 & \text { if } i=1\end{cases}
$$

satisfies $\left\|T^{n}\right\| \rightarrow \infty$, but $\left\|T^{n}(x)\right\| \not \rightarrow \infty$ for all $x$ [6, Example 4]. In [2, pp. 66-68], there is an example of an operator $T$ on Hilbert space satisfying $\left\|T^{n}\right\| \rightarrow \infty$, but $\inf _{n}\left\|T^{n}(x)\right\|=0$ for all $x$.

The object of this note is to show that there is a wide class of Banach spaces which admit operators failing Conjecture 1.1. In fact, by constructing a range of suitable operators, we can impose a reasonable degree of control over the structure of $A_{T}$. Clearly, $A_{T}$ is always radial, in the sense that if $x \in A_{T}$ and $\lambda \neq 0$ then $\lambda x \in A_{T}$. Thus we need only trouble ourselves with what happens to points in the unit sphere $S_{X}$.

We shall consider both real and complex Banach spaces. Recall that if a Schauder basis $\left(e_{i}\right)_{i=1}^{\infty}$ of $X$ is symmetric then there exists an equivalent norm $\|\cdot\|$ on $X$ with the property that

$$
\left\|\sum_{i=1}^{\infty} x_{i} e_{i}\right\|=\left\|\sum_{i=1}^{\infty} \lambda_{i} x_{i} e_{\pi(i)}\right\|
$$

whenever $\pi$ is a permutation of $\mathbb{N}$ and $\left|\lambda_{i}\right|=1$ for all $i$. Such a norm is called symmetric; hereafter, whenever we have a Banach space $X$ with a symmetric basis, we shall assume that $X$ is infinite-dimensional and that the associated norm is symmetric. In addition, we shall say that a subset $E$ of a Banach space is symmetric if $\lambda x \in E$ whenever $x \in E$ and $|\lambda|=1$. Here follows our main result.

Theorem 1.2. Let $X$ have a symmetric basis with norm $\|\cdot\|$ and suppose that $Y \subseteq X$ is a subspace of dimension $d$, where $2 \leq d<\infty$. Moreover, let $E \subseteq S_{Y}$ be closed and symmetric, and let $J$ be a projection of $X$ onto $Y$. Then there exists an operator $R: X \longrightarrow X$ with two properties:

(1) if $J(x) \in E$ then $\left\|R^{n}(x)\right\| \rightarrow \infty$;

(2) if $J(x) \in S_{Y} \backslash E$ then there is a subsequence $\left(R^{n_{i}}(x)\right)$ of $\left(R^{n}(x)\right)$ such that $R^{n_{i}}(x) \rightarrow x$ weakly. 
Of course, we obtain the claim in the abstract by ensuring that $E \subseteq S_{Y}$ in Theorem 1.2 is non-empty and nowhere dense. Roughly speaking, we use the extra dimensions in the complement of $Y$ in $X$ to encode the non-linear information in $E$. To give an idea of what we mean by this, we can compare, at a distance, this encoding of non-linear information to the standard method of producing an operator on Hilbert space with a prescribed spectrum, namely, by arranging a suitable, countable family of eigenvalues. The proof of Theorem 1.2 is spread across sections 2 and 3. We expect that it is possible to generalise Theorem 1.2 to incorporate subsets of $S_{Y}$ of greater topological complexity, but to do so would go beyond the immediate aims of this paper and would unduly complicate our existing proof.

Of course, if $X$ in Theorem 1.2 is complemented in some overspace $Z$ then we obtain a corresponding result about $Z$. In particular, by considering $c_{0}$ or $\ell_{p}, 1 \leq$ $p<\infty$, we can see that any 'classical' Banach space admits an operator $T$ such that $A_{T}$ is non-empty and nowhere dense.

We finish this section by showing that the richness of structure of $A_{T}$, demonstrated by Theorem 1.2, cannot be reproduced in the finite-dimensional setting. In fact, we show that the operator $R$ in Theorem 1.2 cannot be compact in general.

Proposition 1.3. Let $X$ be a Banach space and $T: X \longrightarrow X$ a compact operator. Then there exist finite-codimensional subspaces $Z \subseteq Y \subseteq X$ with the property that

(1) if $x \in X \backslash Y$ then $\left\|T^{n}(x)\right\| \rightarrow \infty$;

(2) if $x \in Y \backslash Z$ then there exists $M>0$ such that $M^{-1} \leq\left\|T^{n}(x)\right\| \leq M$ for every $n$;

(3) if $x \in Z$ then $\left\|T^{n}(x)\right\| \rightarrow 0$.

In particular, if $T$ is compact then $A_{T}$ is simply the complement of a finitecodimensional subspace and, in particular, either empty or dense. Proposition 1.3 is a mild elaboration and generalisation of the behaviour of orbits in finite-dimensional space, stated at the beginning of this note. The result is probably folklore but we sketch a proof of it for completeness.

Sketch proof of Proposition 1.3. First, we assume that $X$ is complex. Using the standard spectral theory of compact operators and the theory of Jordan normal forms, we know that we can find a (possibly empty) sequence of finite-dimensional subspaces $X_{1}, \ldots, X_{n}$, and a finite-codimensional subspace $Z$, all invariant for $T$, such that

$$
X=X_{1} \oplus \ldots \oplus X_{n} \oplus Z .
$$

Moreover, the subspaces $X_{i}$ satisfy the following properties.

(a) each $X_{i}$ has some basis $e_{i, 1}, \ldots, e_{i, m_{i}}$ and associated biorthogonal functionals $f_{i, 1}, \ldots, f_{i, m_{i}}$ on $X_{i}$, such that

(i) $f_{i, k}\left(T\left(e_{i, k}\right)\right)=\lambda_{i}$ for $1 \leq k \leq m_{i}$;

(ii) $f_{i, k-1}\left(T\left(e_{i, k}\right)\right)=1$ for $2 \leq k \leq m_{i}$;

(iii) $f_{i, l}\left(T\left(e_{i, k}\right)\right)=0$ otherwise.

(b) if $x$ is an eigenvector of $T$ with eigenvalue $\lambda$, and $|\lambda| \geq 1$, then $x \in X_{i}$ and $\lambda=\lambda_{i}$ for some $i \leq n$. 
Define $Y=\left[e_{i, 1}\right]_{1 \leq i \leq n,\left|\lambda_{i}\right|=1} \oplus Z$, where $[\cdot]$ denotes norm-closed linear span. To prove the proposition, it is sufficient to show that

(I) $\left\|T^{n}(x)\right\| \rightarrow \infty$ whenever $x \in X_{i} \backslash Y$;

(II) $\left\|T^{n}(x)\right\|=\|x\|$ whenever $x \in X_{i} \cap Y$

(III) $\left\|T^{n}(x)\right\| \rightarrow 0$ whenever $x \in Z$.

First, take $x \in X_{i}$ such that $f_{i, j}(x)=0$ for $j>k$. Using properties (ai)-(aiii), we see that

$$
f_{i, k}(T(x))=\sum_{l=1}^{m_{i}} f_{i, k}\left(T\left(e_{i, l}\right)\right) f_{i, l}(x)=\lambda_{i} f_{i, k}(x)
$$

and $f_{i, j}(T(x))=0$ for $j>k$. Thus, by induction, $f_{i, k}\left(T^{n}(x)\right)=\lambda_{i}^{n} f_{i, k}(x)$. Moreover, if $k>1$ then we calculate

$$
f_{i, k-1}(T(x))=\sum_{l=1}^{m_{i}} f_{i, k-1}\left(T\left(e_{i, l}\right)\right) f_{i, l}(x)=\lambda_{i} f_{i, k-1}(x)+f_{i, k}(x)
$$

and by a second induction we obtain

$$
f_{i, k-1}\left(T^{n}(x)\right)=\lambda_{i}^{n} f_{i, k-1}(x)+n \lambda_{i}^{n-1} f_{i, k}(x) .
$$

Now consider (I). If $x \notin Y$ then in particular $f_{i, k}(x) \neq 0$ for maximal $k \leq m_{i}$. There are two cases. If $\left|\lambda_{i}\right|>1$ then

$$
\left\|f_{i, k}\right\||| T^{n}(x) \| \geq f_{i, k}\left(T^{n}(x)\right)=\left|\lambda_{i}\right|^{n}\left|f_{i, k}(x)\right| \rightarrow \infty .
$$

If instead $\left|\lambda_{i}\right|=1$ then we must have $k \geq 2$, thus

$$
|| f_{i, k-1}|||| T^{n}(x) \| \geq\left|f_{i, k-1}\left(T^{n}(x)\right)\right| \geq n\left|f_{i, k}(x)\right|-\left|f_{i, k-1}(x)\right| \rightarrow \infty .
$$

To see (II), note that the results above give $T^{n}\left(e_{i, 1}\right)=\lambda_{i}^{n} e_{i, 1}$ straightaway. For (III), observe that by property (b) above, we have ensured that the restriction $S$ of $T$ to $Z$ has spectral radius $\alpha<1$. If $x \in Z$ and $\alpha<\beta<1$ then

$$
\left\|T^{n}(x)\right\|=\left\|S^{n}(x)\right\| \leq\left\|S^{n}\right\|\|x\| \leq \beta^{n}\|x\| \rightarrow 0 .
$$

for large enough $n$.

This completes the proof in the complex case. If $X$ is real then we pass to its complexification $X_{\mathbb{C}}$ and consider the compact operator $T_{\mathbb{C}}$, defined by $T_{\mathbb{C}}(x+\mathrm{i} y)=$ $T x+\mathrm{i} T y$.

\section{Local estimates}

Our map $R$ in Theorem 1.2 is going to be a block diagonal operator on $X$. In this section, we build the template for the operators acting on the blocks and gather together some basic estimates. Let $m, T \in \mathbb{N}, \varepsilon>0$ and $Y=\ell_{p}^{T}$, where $4 m \leq T$ and $1 \leq p \leq \infty$. Define the operators $S: Y \longrightarrow Y$ and $F: \mathbb{R} \longrightarrow Y$ by

$$
S(y)=\left(y_{T}, y_{1}, \ldots, y_{T-1}\right)
$$

where $y=\left(y_{1}, \ldots, y_{T}\right)$, and

$$
F(a)=(\underbrace{\varepsilon a, \ldots, \varepsilon a}_{m \text { times }}, \underbrace{-\varepsilon a, \ldots,-\varepsilon a}_{m \text { times }}, 0, \ldots, 0) .
$$


In this way, $S$ can be described as a shift operator and $F$ a 'feed' operator. Let $R: \mathbb{R} \oplus Y \longrightarrow \mathbb{R} \oplus Y$ be defined by $R(a, y)=(a, S(y)+F(a))$. We are interested in the behaviour of $R^{t}(a, 0)$ at time $t \in \mathbb{N}$. We can imagine that $S$ drives an airport baggage carousel and $F$ deposits the passengers' bags onto the moving belt at a fixed set of positions (although some of the bags have 'negative mass'). The absolute mass of bags deposited depends on the value of the first coordinate. Aided by this analogy, we can see that the result of repeated applications of $R$ to the vector $(a, 0)$ can be viewed as the sum of two bumps: one stationary bump of height $\varepsilon a m$ and base width $2 m$, and a moving bump of height $-\varepsilon a m$ and base width again $2 m$. The moving bump's motion is periodic, with period $T$. Let us denote by $P$ the map $(a, y) \mapsto y$.

Lemma 2.1. Let $1 \leq p \leq \infty$. Firstly, if $m \leq t \leq T-m$ then

$$
\left\|P R^{t}(a, 0)\right\| \geq \begin{cases}\left(\frac{2}{p+1}\right)^{p^{-1}} \varepsilon m^{(p+1) p^{-1}}|a| & \text { if } p<\infty \\ \varepsilon m|a| & \text { if } p=\infty .\end{cases}
$$

Secondly, there exists a constant $L$, depending only on $p$, such that at all times $t$ we have

$$
\left\|P R^{t}(a, 0)\right\| \leq \begin{cases}L \varepsilon m^{(p+1) p^{-1}}|a| & \text { if } p<\infty \\ L \varepsilon m|a| & \text { if } p=\infty\end{cases}
$$

and if $t \leq m$ then

$$
\left\|P R^{t}(a, 0)\right\| \leq \begin{cases}L \varepsilon m^{p^{-1}} t|a| & \text { if } p<\infty \\ L \varepsilon t|a| & \text { if } p=\infty\end{cases}
$$

Proof. We estimate the norm of the sum of the standing and moving bumps. If $p=\infty$ we simply measure the absolute height of the sum of the bumps to obtain the values listed above, with $L=1$. From now on, we shall assume that $p<\infty$. Set

$$
L=\left(\frac{2^{p+3}}{p+1}\right)^{\frac{1}{p}}>\left(2+\frac{2^{p+2}+1}{p+1}\right)^{\frac{1}{p}} .
$$

For (2.1), we have

$$
\|\left. P R^{t}(a, 0)\right|^{p} \geq 2 \varepsilon^{p}|a|^{p} \int_{0}^{m} s^{p} \mathrm{~d} s=\frac{2 \varepsilon^{p}|a|^{p}}{p+1} m^{p+1} .
$$

To establish (2.2), we note that the maximum value of the norm is attained when the supports of the standing and moving bumps are disjoint, which occurs if and only if $2 m \leq t \leq T-2 m$. Thus we estimate

$$
\|\left. P R^{t}(a, 0)\right|^{p} \leq 4 \varepsilon^{p}|a|^{p} \int_{0}^{m+1} s^{p} \mathrm{~d} s=\frac{4 \varepsilon^{p}|a|^{p}}{p+1}(m+1)^{p+1} \leq \frac{2^{p+3} \varepsilon^{p}|a|^{p}}{p+1} m^{p+1} .
$$

For (2.3), when $t \leq m$, we note

$$
\begin{aligned}
\left\|P R^{t}(a, 0)\right\|^{p} & \leq 2 \varepsilon^{p}|a|^{p}\left\{(m-t) t^{p}+\int_{0}^{t+1} s^{p} \mathrm{~d} s+\int_{0}^{\frac{t}{2}}(2 s)^{p} \mathrm{~d} s\right\} \\
& =2 \varepsilon^{p}|a|^{p}\left\{(m-t) t^{p}+\frac{(t+1)^{p+1}}{p+1}+\frac{t^{p+1}}{2(p+1)}\right\}
\end{aligned}
$$




$$
\leq\left(2+\frac{2^{p+2}+1}{p+1}\right) \varepsilon^{p} m t^{p}|a|^{p}
$$

In order to build our operator $R$ on a Banach space $X$ with a symmetric basis, we will need to estimate the norms of certain vectors in $X$ with reasonable precision. In order to do this, we combine the estimates of Lemma 2.1 with a result closely based on a theorem of Tzafriri 9. We have altered the statement of the next result to suit our purposes. In what follows, $d(\cdot, \cdot)$ indicates Banach-Mazur distance and, as above, $[\cdot]$ denotes norm-closed linear span.

Proposition 2.2 ([9, Proposition 5]). Let $V$ be a $2^{n}$-dimensional vector space with basis $\left(v_{\sigma}\right)_{\sigma \in G}$, where $G$ is the set of all functions from $\{1, \ldots, n\}$ to $\{-1,1\}$. Suppose that there are constants $K>0$ and $r>2$ such that given scalars $a_{\sigma}, \sigma \in G$, we have

$$
\frac{K^{-1}}{\left(2^{n}\right)^{\frac{1}{s}}}\left(\sum_{\sigma \in G}\left|a_{\sigma}\right|^{s}\right)^{\frac{1}{s}} \leq\left\|\sum_{\sigma \in G} a_{\sigma} v_{\sigma}\right\| /\left\|\sum_{\sigma \in G} v_{\sigma}\right\| \leq \frac{K}{\left(2^{n}\right)^{\frac{1}{r}}}\left(\sum_{\sigma \in G}\left|a_{\sigma}\right|^{r}\right)^{\frac{1}{r}}
$$

where $r^{-1}+s^{-1}=1$. Then there exists $M$, dependent on $K$ and $r$, but independent of $V$ and $n$, with the property that if we define

$$
z_{l}=\sum_{\sigma \in G} \sigma(l) v_{\sigma}
$$

for $1 \leq l \leq n$, then $d\left(\left[z_{l}\right]_{l=1}^{n}, \ell_{2}^{n}\right)<M$.

The proof of the next result closely follows that of [9, Theorem 1], although we note that the assumed symmetry of the norm allows us to bypass the Ramsey arguments which feature in [9]. Tzafriri's notation has also been modified slightly to suit our requirements.

Lemma 2.3. Let $X$ have a normalised symmetric basis $\left(e_{i}\right)_{i=1}^{\infty}$ with conjugate system $\left(e_{i}^{*}\right)_{i=1}^{\infty}$ and symmetric norm $\|\cdot\|$. Then there exists $M>0$ and $p \in\{1,2, \infty\}$, a pairwise disjoint family of finite subsets $F_{n} \subseteq \mathbb{N}, n \in \mathbb{N}$, vectors $z_{l, n}, 1 \leq l \leq n$, supported on $F_{n}$ and permutations $\pi_{n}$ of $F_{n}$ with three properties:

(1) given $n$, if a linear operator $S$ on $X$ satisfies $S\left(e_{i}\right)=e_{\pi_{n}(i)}$ for all $i \in F_{n}$, then $S\left(z_{l, n}\right)=z_{\tau(l), n}$, where $\tau$ is the cycle $(1, \ldots, n)$;

(2) $d\left(\left[z_{l, n}\right]_{l=1}^{n}, \ell_{p}^{n}\right)<M$ for all $n$;

(3) $\pi_{n}$ has order $n$.

Proof. Define

$$
\lambda(n)=\left\|e_{1}+\ldots+e_{n}\right\| \text { and } \mu(n)=\left\|e_{1}^{*}+\ldots+e_{n}^{*}\right\| .
$$

We follow the proof of [9, Theorem 1] in distinguishing three cases.

Case I: for every $n \in \mathbb{N}$ there exists $m_{n} \in \mathbb{N}$ such that $\lambda\left(n m_{n}\right) / \lambda\left(m_{n}\right)<2$. Put $p=\infty$. Set $k_{1}=0$ and, given $k_{n}$, define $k_{n+1}=k_{n}+n m_{n}$. Let

$$
F_{n}=\left\{k_{n}+1, \ldots, k_{n}+n m_{n}\right\}
$$


and define

$$
z_{l, n}=\left(e_{k_{n}+(l-1) m_{n}+1}+\ldots e_{k_{n}+l m_{n}}\right) / \lambda\left(m_{n}\right)
$$

for $1 \leq l \leq n, n \in \mathbb{N}$. Finally, define

$$
\pi_{n}\left(k_{n}+(l-1) m_{n}+r\right)= \begin{cases}k_{n}+l m_{n}+r & \text { if } 1 \leq l<n \text { and } 1 \leq r \leq m_{n} \\ k_{n}+r & \text { if } l=n \text { and } 1 \leq r \leq m_{n} .\end{cases}
$$

It is clear that the $F_{n}$ are pairwise disjoint and properties (11) and (3) hold. Now we prove (2). By the symmetry of the norm, we have $\left\|z_{l, n}\right\|=1$. Since

$$
\begin{aligned}
\max _{l=1}^{n}\left|a_{l}\right| \leq\left\|\sum_{l=1}^{n} a_{l} z_{l, n}\right\| & \leq \max _{l=1}^{n}\left|a_{l}\right|\left\|\sum_{l=1}^{n} z_{l, n}\right\| \\
& \leq \max _{l=1}^{n}\left|a_{l}\right| \frac{\lambda\left(n m_{n}\right)}{\lambda\left(m_{n}\right)} \leq 2 \max _{l=1}^{n}\left|a_{l}\right|
\end{aligned}
$$

for any scalars $a_{1}, \ldots, a_{n}$, we can see that (2) holds for any $M>2$.

Case II: for every $n \in \mathbb{N}$ there exists $m_{n} \in \mathbb{N}$ such that $\mu\left(n m_{n}\right) / \mu\left(m_{n}\right)<2$. Now put $p=1$ and set $k_{n}, F_{n}$ and $\pi_{n}$ exactly as in case I. If we set

$$
z_{l, n}^{*}=\left(e_{k_{n}+(l-1) m_{n}+1}^{*}+\ldots e_{k_{n}+l m_{n}}^{*}\right) / \mu\left(m_{n}\right) .
$$

then we have

$$
\max _{l=1}^{n}\left|a_{l}\right| \leq\left\|\sum_{l=1}^{n} a_{l} z_{l, n}^{*}\right\| \leq 2 \max _{l=1}^{n}\left|a_{l}\right|
$$

just as above. Let $z_{1, n}$ satisfy $\left\|z_{1, n}\right\|=1$ and $z_{1, n}^{*}\left(z_{1, n}\right) \geq \frac{1}{2}$, and have support contained in $\left\{k_{n}+1, k_{n}+m_{n}\right\}$, i.e., the support of $z_{1, n}^{*}$. If we let $S$ be a linear operator satisfying $S\left(e_{i}\right)=e_{\pi_{n}(i)}$ for $i \in F_{n}$, and define $z_{l, n}=S^{l-1}\left(z_{1, n}\right)$ for $1<l \leq n$, then it follows by the symmetry of the norm that $\left\|z_{l, n}\right\|=1$ and $z_{l, n}^{*}\left(z_{l, n}\right)=z_{1, n}^{*}\left(z_{1, n}\right)$ whenever $1 \leq l \leq n$. By design, we have ensured that (1) holds. To check (2), we observe that

$$
\left\|\sum_{l=1}^{n} a_{l} z_{l, n}\right\| \leq \sum_{l=1}^{n}\left|a_{l}\right| \leq 2\left(\sum_{l=1}^{n} \lambda_{l} z_{l, n}^{*}\right)\left(\sum_{k=1}^{n} a_{k} z_{k, n}\right) \leq 4\left\|\sum_{l=1}^{n} a_{l} z_{l, n}\right\|
$$

where $\lambda_{l} a_{l}=\left|a_{l}\right|$ for $1 \leq l \leq n$. Therefore (2) holds whenever $M>4$.

Case III: if neither case I nor case II hold then, following the proof of [9, Theorem 1] in case III, we obtain constants $K>0$ and $r>2$ such that for all $n \in \mathbb{N}$ and scalars $a_{1}, \ldots, a_{n}$, we have

$$
\frac{K^{-1}}{n^{\frac{1}{s}}}\left(\sum_{l=1}^{n}\left|a_{l}\right|^{s}\right)^{\frac{1}{s}} \leq \frac{1}{\lambda(n)}\left\|\sum_{l=1}^{n} a_{l} e_{n+l}\right\| \leq \frac{K}{n^{\frac{1}{r}}}\left(\sum_{l=1}^{n}\left|a_{l}\right|^{r}\right)^{\frac{1}{r}}
$$

where $r^{-1}+s^{-1}=1$. We set $p=2$ and

$$
F_{n}=\left\{2^{n}+1, \ldots, 2^{n+1}\right\} .
$$

Fix $n$ and let $f$ be a bijection from $F=F_{n}$ to $G$, where $G$ is as in Proposition 2.2. Put $v_{\sigma}=e_{f^{-1}(\sigma)}$ for $\sigma \in G$, and let $z_{l}, 1 \leq l \leq n$, be as in Proposition 2.2. Let $\tau$ be the cycle $(1, \ldots, n)$, define a permutation $\hat{\pi}$ on $G$ by $\hat{\pi}(\sigma)=\sigma \circ \tau^{-1}$, and then set 
$\pi=f^{-1} \circ \hat{\pi} \circ f$. We have (3) . If $S$ is an operator on $X$ satisfying $S\left(e_{i}\right)=e_{\pi(i)}$ then we calculate

$$
\begin{aligned}
S\left(z_{l}\right)=S\left(\sum_{\sigma \in G} \sigma(l) v_{\sigma}\right) & =S\left(\sum_{\sigma \in G} \sigma(l) e_{f^{-1}(\sigma)}\right) \\
& =\sum_{\sigma \in G} \sigma(l) e_{f^{-1}(\hat{\pi}(\sigma))} \\
& =\sum_{\sigma \in G} \sigma(l) v_{\hat{\pi}(\sigma)}=\sum_{\sigma \in G}(\sigma \circ \tau)(l) v_{\sigma}=z_{\tau(l)} .
\end{aligned}
$$

Moreover, by construction, we have ensured that $d\left(\left[z_{l}\right]_{l=1}^{n}, \ell_{p}^{n}\right)<M$.

We remark that we can follow the proof of [9, Theorem 1] a little more to show that the subspaces $\left[z_{l, n}\right]_{l=1}^{n}, n \in \mathbb{N}$, are uniformly complemented in $X$, that is, they are the images of a sequence of projections which are uniformly bounded in norm. However, we do not require this particular property of the $\left[z_{l, n}\right]_{l=1}^{n}$.

\section{Proof of Theorem 1.2}

Let $X, Y, E$ and $J$ be as in Theorem 1.2, As $X$ has a symmetric basis, it is isomorphic to its closed, finite-codimensional subspaces. Moreover, $X$ is isomorphic to the space $X_{2}^{d-1}$, which denotes the product $X^{d-1}$, endowed with the norm given by $\left\|\left(x_{1}, \ldots, x_{d-1}\right)\right\|^{2}=\sum_{j=1}^{d-1}\left\|x_{j}\right\|^{2}$. Therefore, by considering a suitable isomorphism, Theorem 1.2 follows from the following proposition.

Proposition 3.1. Whenever $E$ is a closed, symmetric subset of $S_{\ell_{2}^{d}}$, with $2 \leq d<$ $\infty$, then there exists an operator $R: \ell_{2}^{d} \oplus X_{2}^{d-1} \longrightarrow \ell_{2}^{d} \oplus X_{2}^{d-1}$ with two properties:

(1) if $u \in E$ then $\left\|R^{n}(u, x)\right\| \rightarrow \infty$;

(2) if $u \in S_{\ell_{2}^{d}} \backslash E$ then there is a subsequence $\left(R^{n_{i}}(u, x)\right)$ of $\left(R^{n}(u, x)\right)$ such that $R^{n_{i}}(u, x) \rightarrow(u, x)$ weakly.

We shall prove Proposition 3.1 with a sequence of lemmas. The proofs in the real and complex cases are practically identical. First, we consider a map $\rho$ defined on $S_{\ell_{2}^{d}}$ by

$$
\rho(u, v)^{2}=1-|\langle u, v\rangle|^{2}
$$

where $\langle\cdot, \cdot\rangle$ denotes the usual inner product. This function $\rho$ is a pseudometric on $S_{\ell_{2}^{d}}$ which, given $u \in S_{\ell_{2}^{d}}$, identifies the points $\lambda u,|\lambda|=1$. We shall also define

$$
\rho(u, E)=\inf \{\rho(u, v): v \in E\} .
$$

Since $E$ is closed and symmetric, it follows that $\rho(u, E)=0$ if and only if $u \in E$. Given $v \in S_{\ell_{2}^{d}} \backslash E$, we select an orthonormal basis $e_{v, 1}, \ldots, e_{v, d-1}$ of the perpendicular subspace $v^{\perp}$ and define $\Delta_{v}: \ell_{2}^{d} \longrightarrow \ell_{2}^{d-1}$ by

$$
\Delta_{v}(u)=\frac{1}{\rho(v, E)}\left(\left\langle u, e_{v, 1}\right\rangle, \ldots,\left\langle u, e_{v, d-1}\right\rangle\right) .
$$


Obviously, $\left\|\Delta_{v}(u)\right\|_{2}=\rho(u, v) / \rho(v, E)$ whenever $u \in S_{\ell_{2}^{d}}$, where $\|\cdot\|_{2}$ denotes the usual norm on $\ell_{2}^{d-1}$.

Let $W_{n}=\left\{v \in S_{\ell_{2}^{d}}: \rho(v, E) \geq 2^{-n}\right\}$. It is a straightforward matter to show that for each $n$, we can find a $n^{-1}$-net of $S_{\ell_{2}^{d}}$, with respect to $\rho$, which has size of order $n^{2 d-1}$ (or $n^{d-1}$ if we are considering real Banach spaces). Therefore, there exists an integer $K$ such that, for each $n$, there exist vectors

$$
v_{1}^{n}, \ldots v_{K 2^{n(2 d-1)}}^{n} \in W_{n},
$$

with repetitions if necessary, with the property that for any $u \in W_{n}$, we can find $v_{i}^{n}$ satisfying $\rho\left(u, v_{i}^{n}\right) \leq 2^{-n}$.

Lemma 3.2. Let $u \in S_{\ell_{2}^{d}}$. Firstly

$$
\left\|\Delta_{v}(u)\right\|_{2} \leq 2^{n} \quad \text { whenever } v \in W_{n} .
$$

Secondly, if $u \in E$ then

$$
\left\|\Delta_{v}(u)\right\|_{2} \geq 1 \quad \text { whenever } v \in S_{\ell_{2}^{d}} \backslash E .
$$

Finally, if $u \notin E$ then there exists $n_{0}$ with the property that whenever $n>n_{0}$, there exists $i$ in the range $1 \leq i \leq K 2^{n(2 d-1)}$, such that

$$
\left\|\Delta_{v_{i}^{n}}(u)\right\|_{2} \leq 2^{n_{0}+1-n} .
$$

Proof. We only prove (3.3). Fix $n_{0}$ such that $u \in W_{n_{0}}$. For $n>n_{0}$, we can find $v=v_{i}^{n}$ such that $\rho(u, v) \leq 2^{-n}$. Therefore

$$
\rho(v, E) \geq \rho(u, E)-\rho(u, v) \geq 2^{-n_{0}}-2^{-n} \geq 2^{-\left(n_{0}+1\right)}
$$

and $\left\|\Delta_{v}(u)\right\|_{2} \leq 2^{n_{0}+1-n}$.

We take constants $m_{k}, T_{k} \in \mathbb{N}$ and $\varepsilon_{k}>0$. The values of these constants will be chosen in due course. Let $M, p, F_{n}, z_{l, n}$ and $\pi_{n}$ be as in Lemma 2.3. Define

$$
S\left(e_{i}\right)= \begin{cases}e_{\pi_{T_{k}}(i)} & \text { if } i \in F_{T_{k}} \text { for some } T_{k} \\ e_{i} & \text { otherwise }\end{cases}
$$

and extend $\mathrm{S}$ linearly to $X$. As $\|\cdot\|$ is symmetric, $S$ is an isometry. Define operators $S_{k}:\left[z_{l, T_{k}}\right]_{l=1}^{T_{k}} \longrightarrow\left[z_{l, T_{k}}\right]_{l=1}^{T_{k}}$ and $F_{k}: \mathbb{R} \longrightarrow\left[z_{l, T_{k}}\right]_{l=1}^{T_{k}}$ by

$$
S_{k}\left(\sum_{l=1}^{T_{k}} y_{l} z_{l, T_{k}}\right)=\sum_{l=1}^{T_{k}} y_{l} z_{\tau(l), T_{k}}
$$

where $\tau$ is the cycle $\left(1, \ldots, T_{k}\right)$, and

$$
F_{k}(a)=a \varepsilon_{k} \sum_{l=1}^{m_{k}} z_{l, T_{k}}-a \varepsilon_{k} \sum_{l=m_{k}+1}^{2 m_{k}} z_{l, T_{k}} .
$$

Then define $R_{k}$ on $\mathbb{R} \oplus\left[z_{l, T_{k}}\right]_{l=1}^{T_{k}}$ by

$$
R_{k}(a, y)=\left(a, S_{k}(y)+F_{k}(a)\right)
$$

and let $P_{k}(a, y)=y$ for $a \in \mathbb{R}$ and $y \in\left[z_{l, T_{k}}\right]_{l=1}^{T_{k}}$. Let $Q$ and $Q_{j}$ be the standard projections of $\ell_{2}^{d} \oplus X_{2}^{d-1}$ onto $\ell_{2}^{d}$ and onto the $j$ th copy of $X$, respectively. We 
define integers $C_{1}=1$ and $C_{n+1}=C_{n}+K 2^{n(2 d-1)}$ for $n \geq 1$, and set $w_{k}=v_{k+1-C_{n}}^{n}$ whenever $C_{n} \leq k<C_{n+1}$.

Finally, if $u \in \ell_{2}^{d}$ and $x \in X_{2}^{d-1}$, we can define an operator $R$ on $\ell_{2}^{d} \oplus X_{2}^{d-1}$ by

$$
Q R(u, x)=u \quad \text { and } \quad Q_{j} R(u, x)=S Q_{j}(0, x)+\sum_{k=1}^{\infty} \frac{F_{k}\left(\left\langle u, e_{w_{k}, j}\right\rangle\right)}{\rho\left(w_{k}, E\right)}
$$

for $1 \leq j \leq d-1$ and where $e_{w_{k}, j}$ is the $j$ th element of the given basis of $w_{k}^{\perp}$, chosen above.

Of course, it is necessary to choose the constants $m_{k}, T_{k}$ and $\varepsilon_{k}$ so that $R$ is bounded and maps into $X$. First let $m_{1}=1$ and $T_{0}=1$. Then set $T_{k}=\left(5^{d n}+1\right) T_{k-1}$, $m_{k}=T_{k-1}-m_{k-1}$ and

$$
\varepsilon_{k}= \begin{cases}\frac{n}{m_{k}^{(p+1) p^{-1}}} & \text { if } p=1 \text { or } p=2 \\ \frac{n}{m_{k}} & \text { if } p=\infty\end{cases}
$$

whenever $C_{n} \leq k<C_{n+1}$. Our first task is to show that, with respect to these constants, $R$ is a bounded operator mapping into $\ell_{2}^{d} \oplus X_{2}^{d-1}$.

Lemma 3.3. The operator $R$ is bounded and maps into $\ell_{2}^{d} \oplus X_{2}^{d-1}$.

Proof. It is enough to show that $\sum_{k=1}^{\infty} \rho\left(w_{k}, E\right)^{-1} F_{k}\left(\left\langle u, e_{w_{k}, j}\right\rangle\right)$ is absolutely summable for $1 \leq j \leq d-1$. Assume $u \in S_{\ell_{2}^{d}}$. By Lemma 2.3, part 2, we have

$$
M^{-\frac{1}{2}}\|y\|_{p} \leq\left\|\sum_{l=1}^{T_{k}} y_{l} z_{l, T_{k}}\right\| \leq M^{\frac{1}{2}}\|y\|_{p}
$$

where $y=\left(y_{1}, \ldots, y_{T_{k}}\right) \in \ell_{p}^{T_{k}}$ and $\|\cdot\|_{p}$ is the usual norm on $\ell_{p}^{T_{k}}$. We shall assume that $M \geq L$, where $L$ is as in Lemma 2.1. Note that $F_{k}(a)=S_{k}(0)+F_{k}(a)=P_{k} R_{k}(a, 0)$. Therefore, from (2.3) with $t=1$, (3.1), (3.4) and the definition of $\varepsilon_{k}$, we have

$$
\begin{aligned}
\frac{\left\|F_{k}\left(\left\langle u, e_{w_{k}, j}\right\rangle\right)\right\|}{\rho\left(w_{k}, E\right)} & \leq \begin{cases}M^{\frac{3}{2}} \varepsilon_{k} m_{k}^{p^{-1}} \rho\left(w_{k}, E\right)^{-1}\left|\left\langle u, e_{w_{k}, j}\right\rangle\right| & \text { if } p=1,2 \\
M^{\frac{3}{2}} \varepsilon_{k} \rho\left(w_{k}, E\right)^{-1}\left|\left\langle u, e_{w_{k}, j}\right\rangle\right| & \text { if } p=\infty\end{cases} \\
& \leq M^{\frac{3}{2}} n\left\|\Delta_{w_{k}}(u)\right\|_{2} m_{k}^{-1} \\
& \leq M^{\frac{3}{2}} n 2^{n} m_{k}^{-1}
\end{aligned}
$$

whenever $C_{n} \leq k<C_{n+1}$.

From the definitions of $m_{k}$ and $T_{k}$, we obtain

$$
m_{k+1}=T_{k}-m_{k}=T_{k}-T_{k-1}+m_{k-1} \geq 5^{d n} T_{k-1} \geq 5^{d n} m_{k}
$$

whenever $C_{n} \leq k<C_{n+1}$. In particular, $m_{k+1} \geq 5^{d} m_{k}$. Therefore

$$
\begin{aligned}
\sum_{k=1}^{\infty} \frac{\left\|F_{k}\left(\left\langle u, e_{w_{k}, j}\right\rangle\right)\right\|}{\rho\left(w_{k}, E\right)} & \leq \sum_{n=1}^{\infty} \sum_{k=C_{n}}^{C_{n+1}-1} M^{\frac{3}{2}} n 2^{n} m_{k}^{-1} \\
& \leq M^{\frac{3}{2}} m_{1}^{-1} \sum_{n=1}^{\infty} \sum_{k=C_{n}}^{C_{n+1}-1} \frac{n 2^{n}}{5^{d(k-1)}}
\end{aligned}
$$




$$
\begin{aligned}
& \leq M^{\frac{3}{2}} 5^{d} \sum_{n=1}^{\infty} \sum_{k=C_{n}}^{C_{n+1}-1} \frac{n 2^{n}}{5^{d n}} \\
& =M^{\frac{3}{2}} 5^{d} K \sum_{n=1}^{\infty} 2^{n(2 d-1)} \frac{n 2^{n}}{5^{d n}}=M^{\frac{3}{2}} 5^{d} K \sum_{n=1}^{\infty} n\left(\frac{4}{5}\right)^{d n}
\end{aligned}
$$

bearing in mind that $k-1 \geq C_{n}-1 \geq n-1$ whenever $C_{n} \leq k<C_{n+1}$. Hence $R$ is bounded and $R(u, x) \in \ell_{2}^{d} \oplus X_{2}^{d-1}$.

In order to analyse the behaviour of $R^{m}(u, x)$, it will help to consider separately $R^{m}(u, 0)$ and $R^{m}(0, x)$.

Lemma 3.4. Given $(u, x) \in \ell_{2}^{d} \oplus X_{2}^{d-1}$, we have

$$
Q_{j} R^{m}(0, x)=S^{m} Q_{j}(0, x)
$$

and

$$
Q_{j} R^{m}(u, 0)=\sum_{k=1}^{\infty} \frac{P_{k} R_{k}^{m}\left(\left\langle u, e_{w_{k}, j}\right\rangle, 0\right)}{\rho\left(w_{k}, E\right)}
$$

for $1 \leq j \leq d-1$ and for all $m$.

Proof. We proceed by induction. Clearly $Q_{j} R(0, x)=S Q_{j}(0, x)$, so (3.6) holds for $m=1$. If (3.6) holds for some $m \geq 1$ and $R^{m}(0, x)=(0, y)$ then

$$
Q_{j} R^{m+1}(0, x)=Q_{j} R(0, y)=S Q_{j}(0, y)=S Q_{j} R^{m}(0, x)=S^{m+1} Q_{j}(0, x) .
$$

Now

$$
P_{k} R_{k}(a, 0)=S_{k}(0)+F_{k}(a)=F_{k}(a)
$$

and $S Q_{j}(u, 0)=0$, so (3.7) holds for $m=1$. Assume that (3.7) holds for some $m \geq 1$. Suppose that

$$
P_{k} R_{k}^{m}(a, 0)=y=\sum_{l=1}^{T_{k}} y_{l} z_{l, T_{k}} .
$$

By Lemma 2.3, we have ensured that $S(y)=S_{k}(y)$. Furthermore, we observe

$$
\begin{aligned}
P_{k} R_{k}^{m+1}(a, 0)=P_{k} R_{k}\left(R_{k}^{m}(a, 0)\right) & =P_{k} R_{k}(a, y) \\
& =S_{k}(y)+F_{k}(a) \\
& =S(y)+F_{k}(a)=S P_{k} R_{k}^{m}(a, 0)+F_{k}(a) .
\end{aligned}
$$

Therefore, if $R^{m}(u, 0)=(u, z)$ then

$$
\begin{aligned}
Q_{j} R^{m+1}(u, 0) & =Q_{j} R(u, z) \\
& =S Q_{j}(0, z)+\sum_{k=1}^{\infty} \frac{F_{k}\left(\left\langle u, e_{w_{k}, j}\right\rangle\right)}{\rho\left(w_{k}, E\right)} \\
& =S Q_{j} R^{m}(u, 0)+\sum_{k=1}^{\infty} \frac{F_{k}\left(\left\langle u, e_{w_{k}, j}\right\rangle\right)}{\rho\left(w_{k}, E\right)}
\end{aligned}
$$




$$
\begin{aligned}
& =S\left(\sum_{k=1}^{\infty} \frac{P_{k} R_{k}^{m}\left(\left\langle u, e_{w_{k}, j}\right\rangle, 0\right)}{\rho\left(w_{k}, E\right)}\right)+\sum_{k=1}^{\infty} \frac{F_{k}\left(\left\langle u, e_{w_{k}, j}\right\rangle\right)}{\rho\left(w_{k}, E\right)} \\
& =\sum_{k=1}^{\infty} \frac{S P_{k} R_{k}^{m}\left(\left\langle u, e_{w_{k}, j}\right\rangle, 0\right)+F_{k}\left(\left\langle u, e_{w_{k}, j}\right\rangle\right)}{\rho\left(w_{k}, E\right)} \\
& =\sum_{k=1}^{\infty} \frac{P_{k} R_{k}^{m+1}\left(\left\langle u, e_{w_{k}, j}\right\rangle, 0\right)}{\rho\left(w_{k}, E\right)}
\end{aligned}
$$

as required.

The consequence of Lemma 3.4 is that we can split the analysis of $R^{m}(u, x)$ into two parts: the 'shift' and the 'perturbation'. First, we examine the behaviour of the shift.

Lemma 3.5. Given $x \in X_{2}^{d-1}$, we have $\left\|R^{m}(0, x)\right\|=\|(0, x)\|$ for all $m$. Moreover, $R^{T_{k}}(0, x) \stackrel{w}{\rightarrow}(0, x)$.

Proof. Given (3.6) and the fact that $S$ is an isometry, the first assertion is trivial. Now consider the weak convergence. Let $f \in X^{*}$ with $\|f\|=1, \varepsilon>0$ and $1 \leq j \leq$ $d-1$. If $Q_{j}(0, x)=\sum_{i=1}^{\infty} x_{i} e_{i}$, we take $k \in \mathbb{N}$ such that

$$
\left\|\sum_{l=k+1}^{\infty} \sum_{i \in F_{T_{l}}} x_{i} e_{i}\right\|<\varepsilon .
$$

Since $T_{l}$ divides $T_{r}$ whenever $l \leq r$, we can see that $\pi_{T_{l}}^{T_{r}}$ is the identity for such $l$. Therefore, if $r \geq k$, we estimate

$$
\begin{aligned}
\left|f\left(Q_{j} R^{T_{k}}(0, x)-Q_{j}(0, x)\right)\right| & =\left|f\left(S^{T_{r}} Q_{j}(0, x)-Q_{j}(0, x)\right)\right| \\
& =\left|f\left(\sum_{l=r+1}^{\infty} \sum_{i \in F_{T_{l}}} x_{i} e_{\pi_{T_{l}}^{T_{r}}(i)}-\sum_{l=r+1}^{\infty} \sum_{i \in F_{T_{l}}} x_{i} e_{i}\right)\right| \\
& \leq 2\left\|\sum_{l=r+1}^{\infty} \sum_{i \in F_{T_{l}}} x_{i} e_{i}\right\| \\
& \leq 2\left\|\sum_{l=k+1}^{\infty} \sum_{i \in F_{T_{l}}} x_{i} e_{i}\right\|<2 \varepsilon
\end{aligned}
$$

by symmetry of the norm. Thus $Q_{j} R^{T_{k}}(0, x) \stackrel{w}{\rightarrow} Q_{j}(0, x)$ whenever $1 \leq j \leq d-1$. The weak convergence of $R^{T_{k}}(0, x)$ to $(0, x)$ follows.

Now we analyse the behaviour of the perturbation. Ultimately, it is the perturbation that drives the behaviour of the system as a whole.

Lemma 3.6. If $u \in E$ then

$$
\left\|R^{m}(u, 0)\right\| \rightarrow \infty
$$


On the other hand, if $u \in S_{\ell_{2}^{d}} \backslash E$ then there exists $k_{n}$ in the range $C_{n} \leq k_{n}<C_{n+1}$ with the property that

$$
\left\|R^{T_{k_{n}-1}}(u, 0)-(u, 0)\right\| \rightarrow 0 .
$$

Proof. Let $u \in E$ and suppose that $p=1$ or $p=2$. Assume that $m_{k} \leq m<$ $T_{k}-m_{k}=m_{k+1}$ and $C_{n} \leq k<C_{n+1}$. Then by (2.1), (3.2), (3.4), (3.7) and the definition of $\varepsilon_{k}$, we have

$$
\begin{aligned}
\left\|R^{m}(u, 0)\right\|^{2} & \geq \sum_{j=1}^{d-1}\left\|Q_{j} R^{m}(u, 0)\right\|^{2} \\
& \geq \sum_{j=1}^{d-1}\left(\frac{\left\|P_{k} R_{k}^{m}\left(\left\langle u, e_{w_{k}, j}\right\rangle, 0\right)\right\|}{\rho\left(w_{k}, E\right)}\right)^{2} \\
& \geq \sum_{j=1}^{d-1} M^{-1}\left(\frac{2}{p+1}\right)^{2 p^{-1}} \varepsilon_{k}^{2} m_{k}^{2(p+1) p^{-1}}\left(\frac{\left|\left\langle u, e_{w_{k}, j}\right\rangle\right|}{\rho\left(w_{k}, E\right)}\right)^{2} \\
& =M^{-1}\left(\frac{2}{p+1}\right)^{2 p^{-1}} n^{2} \sum_{j=1}^{d-1}\left(\frac{\left|\left\langle u, e_{w_{k}, j}\right\rangle\right|}{\rho\left(w_{k}, E\right)}\right)^{2} \\
& \geq M^{-1}\left(\frac{2}{p+1}\right)^{2 p^{-1}} n^{2}
\end{aligned}
$$

If $u \in E$ and $p=\infty$ then similarly, we obtain

$$
\left\|R^{m}(u, 0)\right\|^{2} \geq M^{-1} n^{2} .
$$

Either way, $\left\|R^{m}(u, 0)\right\| \rightarrow \infty$.

Instead, if $u \in S_{\ell_{2}^{d}} \backslash E$ then by (3.3) , for every $n>n_{0}$ there exists $k_{n}$ in the range $C_{n} \leq k_{n}<C_{n+1}$, such that

$$
\left\|\Delta_{w_{k_{n}}}(u)\right\|_{2} \leq 2^{n_{0}+1-n}
$$

By (2.2), (3.4) and the definition of $\varepsilon_{k}$, we have

$$
\begin{aligned}
\left\|P_{k_{n}} R_{k_{n}}^{T_{k_{n}-1}}\left(\left\langle u, e_{w_{k_{n}}, j}\right\rangle, 0\right)\right\| & \leq \begin{cases}M^{\frac{3}{2}} \varepsilon_{k_{n}} m_{k_{n}}^{(p+1) p^{-1}}\left|\left\langle u, e_{w_{k_{n}}, j}\right\rangle\right| & \text { if } p=1,2 \\
M^{\frac{3}{2}} \varepsilon_{k_{n}} m_{k_{n}} \mid\left\langle u, e_{w_{k_{n}}, j}\right\rangle & \text { if } p=\infty\end{cases} \\
& \leq M^{\frac{3}{2}} \rho\left(w_{k_{n}}, E\right) n 2^{n_{0}+1-n} .
\end{aligned}
$$

Then we notice that if $r \leq k_{n}-1$, we have

$$
\left\|P_{r} R_{r}^{T_{k_{n}-1}}\left(\left\langle u, e_{w_{r}, j}\right\rangle, 0\right)\right\|=0
$$

because $R_{r}^{T_{r}}$ is the identity and $T_{r}$ divides $T_{k_{n}-1}$ whenever $r \leq k_{n}-1$. Now we have to estimate $\left\|P_{r} R_{r}^{T_{k_{n}-1}}\left(\left\langle u, e_{w_{r}, j}\right\rangle, 0\right)\right\|$ for $r \geq k_{n}+1$. If $r \geq k_{n}+1$ then from (3.5), we have

$$
m_{r} \geq 5^{d\left(r-\left(k_{n}+1\right)\right)} m_{k_{n}+1} \geq 5^{d\left(r-\left(k_{n}+1\right)\right)} 5^{d n} T_{k_{n}-1} .
$$


Take $l \geq n$ such that $C_{l} \leq r<C_{l+1}$. Again we assume that $M \geq L$, with $L$ as in Lemma 2.1. We apply (2.3), (3.1) and (3.4) to obtain

$$
\begin{aligned}
& || P_{r} R_{r}^{T_{k_{n}-1}}\left(\left\langle u, e_{w_{r}, j}\right\rangle, 0\right) \| \\
\leq & \begin{cases}M^{\frac{3}{2}} \varepsilon_{r} m_{r}^{p^{-1}} T_{k_{n}-1}\left|\left\langle u, e_{w_{r}, j}\right\rangle\right| & \text { if } p=1,2 \\
M^{\frac{3}{2}} \varepsilon_{r} T_{k_{n}-1}\left|\left\langle u, e_{w_{r}, j}\right\rangle\right| & \text { if } p=\infty\end{cases} \\
\leq & M^{\frac{3}{2}} T_{k_{n}-1} \frac{\rho\left(w_{r}, E\right) l 2^{l}}{m_{r}} \\
\leq & M^{\frac{3}{2}} \rho\left(w_{r}, E\right) \frac{l 2^{l}}{5^{d\left(r-\left(k_{n}+1\right)\right)} 5^{d n}} \\
\leq & M^{\frac{3}{2}} \rho\left(w_{r}, E\right) \frac{l 2^{l}}{5^{d n} 5^{d(l-n)}} \text { since } l-n \leq r-\left(k_{n}+1\right) \\
= & M^{\frac{3}{2}} \rho\left(w_{r}, E\right) \frac{l 2^{l}}{5^{d l}}
\end{aligned}
$$

Combining (3.7) with (3.8), (3.9) and (3.10) gives

$$
\begin{aligned}
\left\|Q_{j} R^{T_{k_{n}-1}}(u, 0)\right\| & \leq \sum_{r=1}^{\infty} \frac{\left\|P_{r} R_{r}^{T_{k_{n}-1}}\left(\left\langle u, e_{w_{r}, j}\right\rangle, 0\right)\right\|}{\rho\left(w_{r}, E\right)} \\
& =\sum_{r=k_{n}}^{\infty} \frac{\left\|P_{r} R_{r}^{T_{k_{n}-1}}\left(\left\langle u, e_{w_{r}, j}\right\rangle, 0\right)\right\|}{\rho\left(w_{r}, E\right)} \\
& =M^{\frac{3}{2}} n 2^{n_{0}+1-n}+\sum_{r=k_{n}+1}^{\infty} \frac{\left\|P_{r} R_{r}^{T_{k_{n}-1}}\left(\left\langle u, e_{w_{r}, j}\right\rangle, 0\right)\right\|}{\rho\left(w_{r}, E\right)} \\
& \leq M^{\frac{3}{2}} n 2^{n_{0}+1-n}+M^{\frac{3}{2}} \sum_{l=n}^{\infty} K 2^{l(2 d-1)} \frac{l 2^{l}}{5^{d l}} \\
& =M^{\frac{3}{2}} n 2^{n_{0}+1-n}+M^{\frac{3}{2}} K \sum_{l=n}^{\infty} l\left(\frac{4}{5}\right)^{d l} \\
& \rightarrow 0
\end{aligned}
$$

as $n \rightarrow \infty$. This concludes the proof.

Proof of Proposition 3.1. Let $u \in E$. By Lemmas 3.5 and 3.6 we have

$$
\left\|R^{m}(u, x)\right\| \geq\left\|R^{m}(u, 0)\right\|-\left\|R^{m}(0, x)\right\|=\left\|R^{m}(u, 0)\right\|-\|(0, x)\| \rightarrow \infty
$$

as $m \rightarrow \infty$.

Now suppose $u \in S_{\ell_{2}^{d}} \backslash E$. Again by Lemmas 3.5 and 3.6, we can pick suitable $k_{n}$ such that

$$
R^{T_{k_{n}-1}}(u, x)=R^{T_{k_{n}-1}}(u, 0)+R^{T_{k_{n}-1}}(0, x) \stackrel{w}{\rightarrow}(u, 0)+(0, x)=(u, x) .
$$


If $X=c_{0}$ or $X=\ell_{p}, 1 \leq p<\infty$, then we can simplify the proof of Theorem 1.2 by replacing the $z_{l, n}$ with unit vectors and replacing the corresponding $\pi_{n}$ with cycles. Since there is a Banach space with a symmetric basis but containing no isomorphic copy of $c_{0}$ or $\ell_{p}, 1 \leq p<\infty$, [3], it is not possible to obtain Theorem [1.2 by proving it in the cases $X=c_{0}$ and $X=\ell_{p}$, and then appealing to complemented subspaces.

\section{Problems}

Since the operators constructed in this note rely fundamentally on permutations of basis vectors, it makes sense to pose the following question.

Problem 4.1. If $X$ is a Banach space with an unconditional basis, does there exist an operator $T$ on $X$ with the property that $\left\|T^{n}(x)\right\| \rightarrow \infty$ for some $x \in X$, and $\left\|T^{n} y\right\| \not \rightarrow \infty$ for all $y$ in a non-empty, open subset of $X$ ?

Also, since the operators which feature above cannot be compact, by Proposition 1.3, the next question seems natural to us.

Problem 4.2. Is it possible to find a sum $I+T$, where $T$ is compact, which satisfies the properties given in the abstract? In particular, does the Argyros-Haydon space [1] admit such an operator?

If no sum $I+T, T$ compact, satisfies the properties given in the abstract, then this suggests to us that some kind of unconditional structure is necessary in order to construct such operators.

Finally, we make a remark about the title of this note. The operator $R$ constructed above can be viewed as a machine which acts on a countable family of disjoint cycles. This family of disjoint cycles can be seen as a countable directed graph. We speculate that it may be possible to construct operators with other interesting properties by basing them on more complicated directed graphs.

\section{REFERENCES}

1. S. A. Argyros and R. G. Haydon, A hereditarily indecomposable $\mathscr{L}_{\infty}$-space that solves the scalar-plus-compact problem. Preprint. http://arxiv.org/abs/0903.3921

2. B. Beauzamy, Introduction to operator theory and invariant subspaces, North-Holland Mathematical Library Vol. 42, North-Holland, Amsterdam (1988).

3. T. Figiel and W. B. Johnson, A uniformly convex Banach space which contains no $\ell_{p}$. Composito Math. 29 (1977), 179-190.

4. V. Müller, Orbits, weak orbits and local capacity of operators. Integral Equations Operator Theory 41 (2001), 230-253.

5. V. Müller, Orbits of Operators, in Advanced courses of mathematical analysis I, Cadiz (2004).

6. V. Müller and J. Vršovský, Orbits of linear operators tending to infinity. Rocky Mountain J. Math. 39 (2009), 219-230.

7. G. Prăjitură, The geometry of an orbit. Preprint.

8. S. Rolewicz, On orbits of elements. Studia Math. 32 (1969), 17-22.

9. L. Tzafriri, On Banach spaces with unconditional bases. Israel J. Math. 17 (1974), 84-93. 
Institute of Mathematics of the AS CR, Žitná 25, CZ - 11567 Praha 1, Czech REPUBLIC

E-mail address: hajek@math.cas.cz

E-mail address: smith@math.cas.cz 